\title{
Establishing quantitative structure tribo-ability relationship model using Bayesian regularization neural network
}

\author{
Xinlei GAO ${ }^{1}$, Kang $\mathrm{DAI}^{2,}{ }^{2,}$, Zhan WANG ${ }^{1}$, Tingting $\mathrm{WANG}^{1}$, Junbo $\mathrm{HE}^{3}$ \\ ${ }^{1}$ School of Chemical and Environmental Engineering, Wuhan Polytechnic University, Wuhan 430023, China \\ ${ }^{2}$ College of Pharmacy, South-Central University for Nationalities, Wuhan 430074, China \\ ${ }^{3}$ College of Food Science and Engineering, Wuhan Polytechnic University, Wuhan 430023, China \\ Received: 11 December 2015 / Revised: 19 January 2016 / Accepted: 22 January 2016 \\ C The author(s) 2016. This article is published with open access at Springerlink.com
}

\begin{abstract}
Quantitative structure-activity relationship methods are used to study the quantitative structure triboability relationship (QSTR), which refers to the tribology capability of a compound from the calculation of structure descriptors. Here, we used the Bayesian regularization neural network (BRNN) to establish a QSTR prediction model. Two-dimensional (2D) BRNN-QSTR models can flexibly and easily estimate lubricant-additive antiwear properties. Our results show that electron transfer and heteroatoms (such as S, P, O, and N) in a lubricant-additive molecule improve the antiwear ability. We also found that molecular connectivity indices are good descriptors of 2D BRNN-QSTR models.
\end{abstract}

Keywords: quantitative structure tribo-ability relationship; Bayesian regularization neural network; lubricant additive; antiwear

\section{Introduction}

To find effective tribological materials suitable for use under different working conditions, numerous experiments need to be performed, including modifying or synthesizing novel potential materials, testing their tribological performance, and screening several compounds. However, we still largely rely on luck to find an effective lubricant. This approach is inefficient because the entire development process lacks theoretical guidance, resulting in a lack of direction in the development process. Finding a compound with good tribological properties from numerous possible structures by a random search and/or based on experience is an inefficient use of time and resources.

It is well known that the physical, chemical, biological, and tribological properties of materials are determined by their structures. However, studies on systematic and scientific methods for linking structures

* Corresponding author: Kang DAI.

E-mail: kangdai1688@163.com and tribological properties of compounds are lacking [1-5]. Furthermore, it remains unclear whether the same principle relates the structures of materials to their tribological properties. Because the structureproperty relationship is well established in chemistry, organic chemical properties can be predicted from the structure of a molecule. In 1962, Hansch founded quantitative structure-activity relationship (QSAR) research methods [6] (REF). In QSAR, effective quantitative calculations or approximation methods are used to establish mathematical relationships between a compound's physicochemical properties and structural parameters (descriptors) [7] for predicting these properties and determining influencing factors. QSAR methods are based on the quantification of the structural characteristics of a compound. A wide range of QSAR descriptors have been developed, including twodimensional (2D) and three-dimensional (3D) structure descriptors $[8,9]$. Based on the principle of QSAR, we developed quantitative structure tribo-ability relationships (QSTRs) [10, 11]; QSTRs apply a QSAR method to identify relationships between structures and 
tribological properties and predict the tribological properties of compounds. The aim here is to provide a theoretical framework for designing a lubricant, improving the efficiency of the lubricant design, and reducing the development cost.

We studied the relationship between evaluation of an infrared vibration-based (EVA) descriptors and tribological properties of several compounds [10] and found a strong correlation between them. The predicted results are consistent with experimental findings, and quantitative calculations confirmed the following. (1) The antiwear mechanism is related to load, and thus, we should consider the effect of load on wear. (2) Elements S and P can significantly improve tribological properties. (3) Minimum carbon chain length is needed for tribological additives, but simply extending the carbon chain may not be effective in obtaining the best antiwear additives. To accurately identify the contribution of the chain length of additives to tribological properties, a topological description of molecular geometry parameters should be considered, as well as EVA descriptors. (4) Finally, the addition of an aromatic ring or double bonds of compounds to improve tribological properties is not necessary.

We used a back-propagation neural network (BPNN)-QSTR model to estimate lubricant-additive antiwear properties [11]. The results indicate that (1) under all loads, the stability of the chemical properties of lubricant additives improves the antiwear performance; and (2) four processes are presumed to occur in friction: diffusion, adsorption, conformation change, and reaction. The contributions of these four processes are different under different loads. Under low loads, the contributions of diffusion and adsorption are dominant. Under intermediate and high loads, molecules become flexible, and the contributions of conformation and tribo-reaction become more prominent.

In QSTR, we experience a crucial problem: which method to adopt for establishing a prediction model. Several machine learning methods such as multiple linear regression, partial least-squares (PLS) method, neural networks, and support vector machines can be used in QSAR. However, in a nonlinear process model, only neural networks are compatible. Therefore, we used a neural network approach to construct a QSTR model. In a previous study [11], we selected BPNN to establish a QSTR model. In this study, we adopted the Bayesian regularization neural network (BRNN) to establish the model. BRNN is a type of a BPNN model with Levenberg-Marquardt optimization and Bayesian learning algorithms. BPNN is the most frequently used neural network for predicting the structure-activity relationship, but it has some drawbacks, such as there is no assurance of finding the global minimum and over-fitting. BRNN, as an updated version of BPNN, minimizes a combination of squared errors and weights and then determines the correct combination to produce a well-generalized neural network model [12]; this process is called Bayesian regularization. Moreover, BRNN can avoid over-fitting in neural networks. Levenberg-Marquardt optimization can run through several feasible parameters to effectively find neural network models that are close to the global minimum. Therefore, BRNN is a robust model with good data fitting and generalization ability.

In this study, we apply BRNN to study the tribological QSAR and estimate the antiwear properties of lubricant additives for determining the material tribology capability from the calculation of structural descriptors.

\section{Methods}

\subsection{Chemicals and data}

Thirty-six compounds, including benzoxazole, benzimidazole, benzothiazole, dihydrothiazole piperazine, and piperidine, were used as lubricant additives, and their tribological data were examined [13]. Friction tests were performed using a four-ball machine tester. Balls were made of GCr 15 (AISI52100) bearing steel with a hardness of 59-61 HRc. All tests were performed in an ambient environment at $20{ }^{\circ} \mathrm{C}$. The rotational velocity, $v$, was $1,450 \mathrm{r} / \mathrm{min}$, and normal loads, $L$, of $196 \mathrm{~N}, 294 \mathrm{~N}$, and $392 \mathrm{~N}$ were applied for $30 \mathrm{~min}$, respectively. Liquid paraffin was used as the base stock. Worn and rubbed surfaces of three bottom steel ball specimens were examined using an optical microscope equipped with a scale used to measure the wear scar diameter. Each test was performed twice, and the measured size of the diameter was an average 
of the scar diameters of six bottom balls' in these two tests $[10,11]$.

All structures were inputted into SYBYL, and their minimum-energy 3D conformations were generated using the standard Tripos method. The tribological data contained the wear scar area of compounds under three loads: 196, 294, and $392 \mathrm{~N}$ [11]. To determine the antiwear ability of molecules at their optimal antiwear concentration, WS was calculated using the following equation:

$$
\mathrm{WS}=\log _{10} \frac{S_{0}^{3 / 2} \times \mathrm{MW}}{S^{3 / 2} \times \mathrm{Conc}}
$$

where WS represents the ability of compounds to decrease the wear scar area under three different loads. $S_{0}$ is the negative control, which is the wear scar area formed under pure base paraffin oil, $S$ is the wear scar area formed with a lubricant additive, MW is the molecular weight of the compound, and Conc is the concentration of the additive that gives the best lubrication [11].

The QSTR descriptors include quantum descriptors, 2D topological descriptors, and 3D Jurs descriptors. Quantum descriptors [11] include the energy of the highest occupied molecular orbital and lowest unoccupied molecular orbital (LUMO), heat of formation, dipole, quadrupole, and other descriptors, which were calculated using the Discovery Studio software. BRNN models were established using the three types of descriptors. The establishment and validation of a neural model were completed using MATLAB. To determine the performance of a BRNNQSTR model, the common regression coefficient $R^{2}$ was calculated, and the cross-validation correlation coefficient $r^{2}$ (leave-one-out cross-validation method, LOO) was employed as a train set. $r^{2}$ indicates the predictive ability or robustness of models for external samples. For samples in test sets, such as $r^{2}$, we named $q^{2}$ to evaluate the predictive ability. $r^{2}$ and $q^{2}$ were calculated using the following equation [10, 11, 14-16]:

$$
q^{2}\left(r^{2}\right)=\frac{\sum_{i}\left(\operatorname{pred}_{i}-\mathrm{obs}_{i}\right)^{2}}{\sum_{i}\left(\mathrm{obs}_{i}-\overline{\mathrm{obs}}\right)^{2}}
$$

where pred is the predicted value and obs is the observed value. We also calculated the sensitivity of each variable in a neural network model to identify the effect of parameter changes on WS. For providing a rough estimation of the effect of parameters on the antiwear performance in the model, the sensitivity $[10,11,14-16]$ is calculated by

$$
\text { Sensitivity } \sim \text { Mean }\left(\frac{\Delta \text { Prediction }}{\Delta \text { Index }}\right)
$$

where $\Delta$ Prediction is the change in the prediction value and $\Delta$ Index is the change in variable parameters. All statistical parameters of these processes were obtained by self-programming.

\section{Results}

\subsection{BRNN-QSTR models}

The key parameter of BRNN models is the number of hidden layer neurons. Because the compound sample is only 36 and the number of hidden layer neurons is high, model over-fitting occurs. Therefore, we limited the number of hidden layer neurons to six (less than $20 \%$ of the total sample). After screening, the number of hidden layer neurons for optimal LOO validation performance was three or four. This model achieves a balance between lack-of-fitting and over-fitting conditions.

The results of the LOO validation of the BRNN models with the three descriptors are shown in Table 1. In Table 1 , because $q^{2}$ of the BRNN models with the three types of load was greater than 0.9 , the BRNN models with the topological descriptors of 2D-QSTR have good prediction performance. However, the performance of the BRNN-QSTR models with 3D Jurs descriptors is poor because all of the three $q^{2}$ values are less than 0.1 , which indicates that 3D Jurs descriptors do not predict the wear scar data well. The performance of the BRNN-QSTR models with the quantum descriptors is reasonable. The $q^{2}$ of the model with $196 \mathrm{~N}$ is greater than 0.7 , while the $q^{2}$ values of the models with the other two loads are about 0.5 , indicating the model prediction performance is slightly poor at high loads. In addition, we used another BRNN-QSTR model with quantum descriptors using the same data with a different self-programming procedures, and found that the models with quantum descriptors were good 
Table 1 Leave-one-out cross-validation for BRNN models with topology, 3D Jurs, and quantum descriptors.

\begin{tabular}{cccc}
\hline Load (N) & $\boldsymbol{q}^{2}$ (Topology) & $\boldsymbol{q}^{2}$ (Jurs_3D) & $\boldsymbol{q}^{2}$ (Quantum) \\
\hline 196 & 0.9092 & -0.2093 & 0.7074 \\
294 & 0.9036 & 0.0799 & $0.5460^{*}$ \\
392 & 0.9222 & 0.0831 & $0.5080^{*}$ \\
\hline
\end{tabular}

*Four hidden layer neurons, the rest have three.

( $q^{2}$ of about 0.85$)$, which indicates that the predictability of BRNN-QSTR models with the quantum descriptors is good [17]. However, in follow-up work, we found that the program tended to over-fit the data. Despite the challenges, the above model can be used to predict trends in wear. We further adjusted the method and the data were reprogrammed, and new models with the quantum descriptors were obtained. Although the $q^{2}$ value was not good compared with our previous results, this model does not over-fit the data. Both of the models with the quantum descriptors are acceptable for modeling and predicting tribological problems.

\subsection{Accuracy of the BRNN-QSTR models}

To further analyze the accuracy of the BRNN prediction model, the $r^{2}$ value was calculated using Eq. (2). According to the results in Section 3.1, we established BRNN models to predict the scar data with topology (2D) and quantum descriptors. In each model, a training set of 32 samples and a test set of 4 samples were constructed. In Table 2, the predictions of the 2D-QSTR model are acceptable (the $r^{2}$ values were more than 0.9), but the predictions of the QSTR models with quantum descriptors are poor (the $r^{2}$ values were less than 0.9). Figures 1-3 show the antiwear fitting diagram of the experimental and predicted values in the 2D-QSTR model and the quantum descriptors of the QSTR model with different loads, and the fitting

Table 2 Prediction performance of BRNN models with topology and quantum descriptors.

\begin{tabular}{ccc}
\hline Load $(\mathbf{N})$ & $\boldsymbol{r}^{2}$ (Topology) & $\boldsymbol{r}^{2}$ (Quantum) \\
\hline 196 & 0.9717 & 0.7530 \\
294 & 0.9630 & $0.8728^{*}$ \\
392 & 0.9490 & $0.8199^{*}$ \\
\hline
\end{tabular}

*Four hidden layer neurons, the rest have three. of the two types of model. In Figs. 1-3, the horizontal axis is the observed or experimental scale of antiwear; and the vertical axis shows the predicted scale of antiwear in the models.

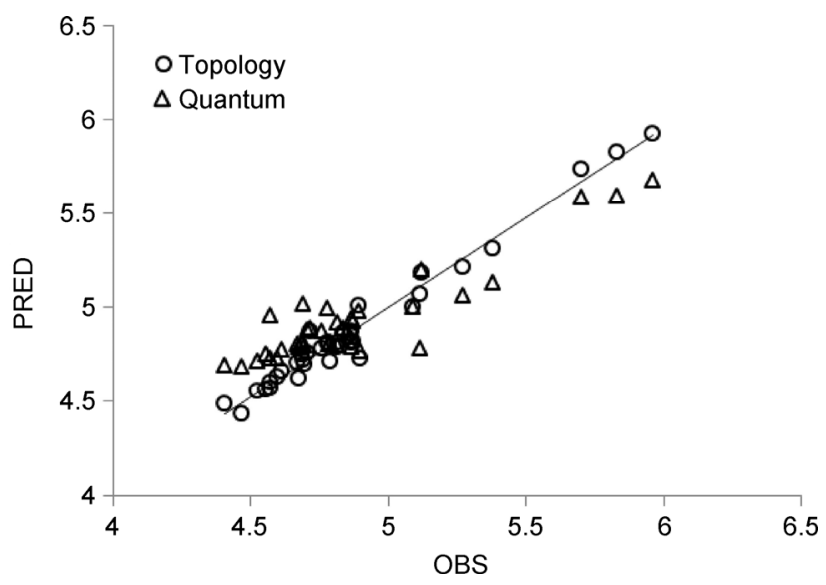

Fig. 1 Predicted (PRED) vs observed (OBS) values of antiwear data under $196 \mathrm{~N}$

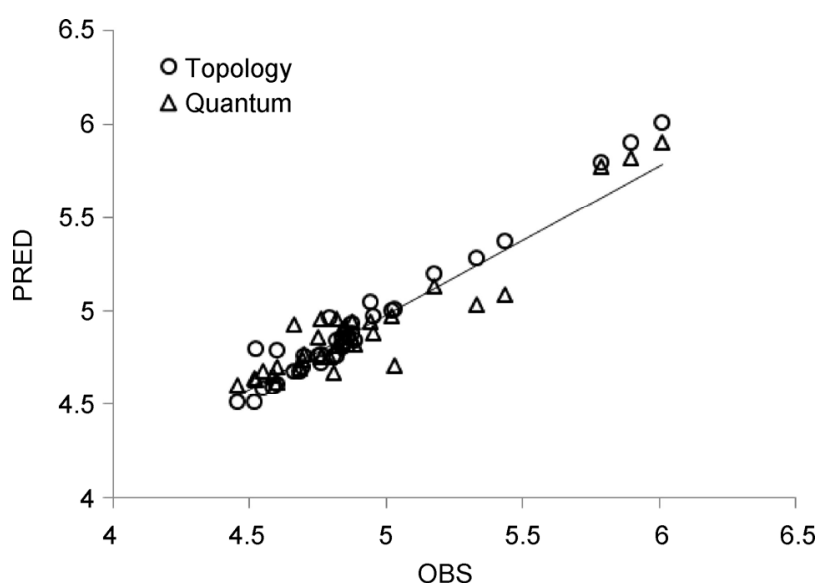

Fig. 2 Predicted (PRED) vs observed (OBS) values of antiwear data under $294 \mathrm{~N}$.

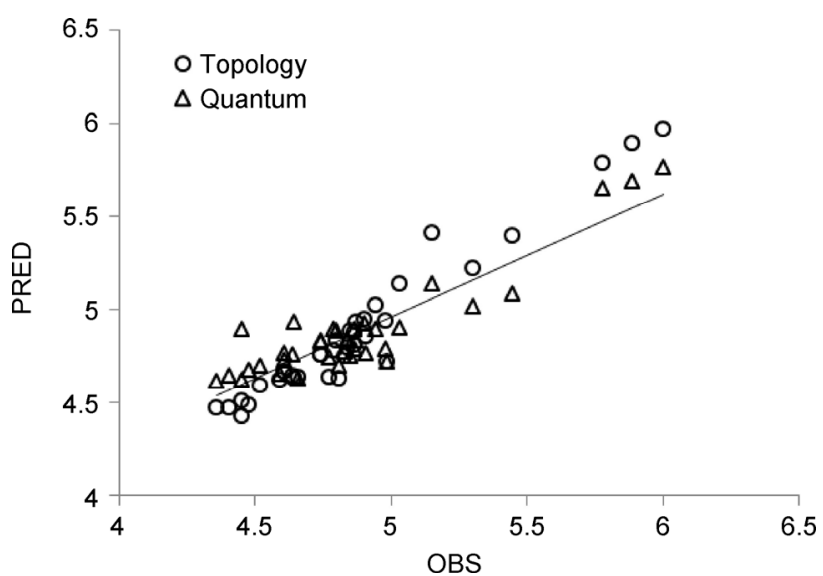

Fig. 3 Predicted (PRED) vs observed (OBS) values of antiwear data under $392 \mathrm{~N}$. 


\subsection{Sensitivity of the descriptors in the BRNN- QSTR model}

Figures 4-6 show the sensitivities of the descriptors in the 2D-QSTR model and the quantum descriptors in the QSTR model, which estimates the contribution of the QSTR descriptors to the antiwear and helps us explore the mechanisms of the lubricant processes.

\section{Discussion}

\subsection{Characteristics of the BRNN-QSTR models}

If the BPNN model achieves high prediction accuracy, then it may be over-fitting, which compromises its ability to predict compounds excluded in the training set. The regularization technique can improve the generalizability of the network. The network training objective function $F$ can be expressed as $F=\alpha E_{\mathrm{W}}+$ $\beta E_{\mathrm{D}}$, where $E_{\mathrm{W}}$ is the weighted sum of squares, $E_{\mathrm{D}}$ is the residual sum of squares of the network response and the target value, and $\alpha$ and $\beta$ are objective function parameters (regularization parameters). The relative values of $\alpha$ and $\beta$ determine if the network training focus reduces the output residuals or decreases the weighting coefficient of the network. Reducing the output residuals enhances the fitting ability of the neural network, while decreasing the weighting coefficient of the network improves the generalizability. The main difficulty in implementing regularization techniques is choosing the appropriate objective function parameters (Bayesian statistics) that are used

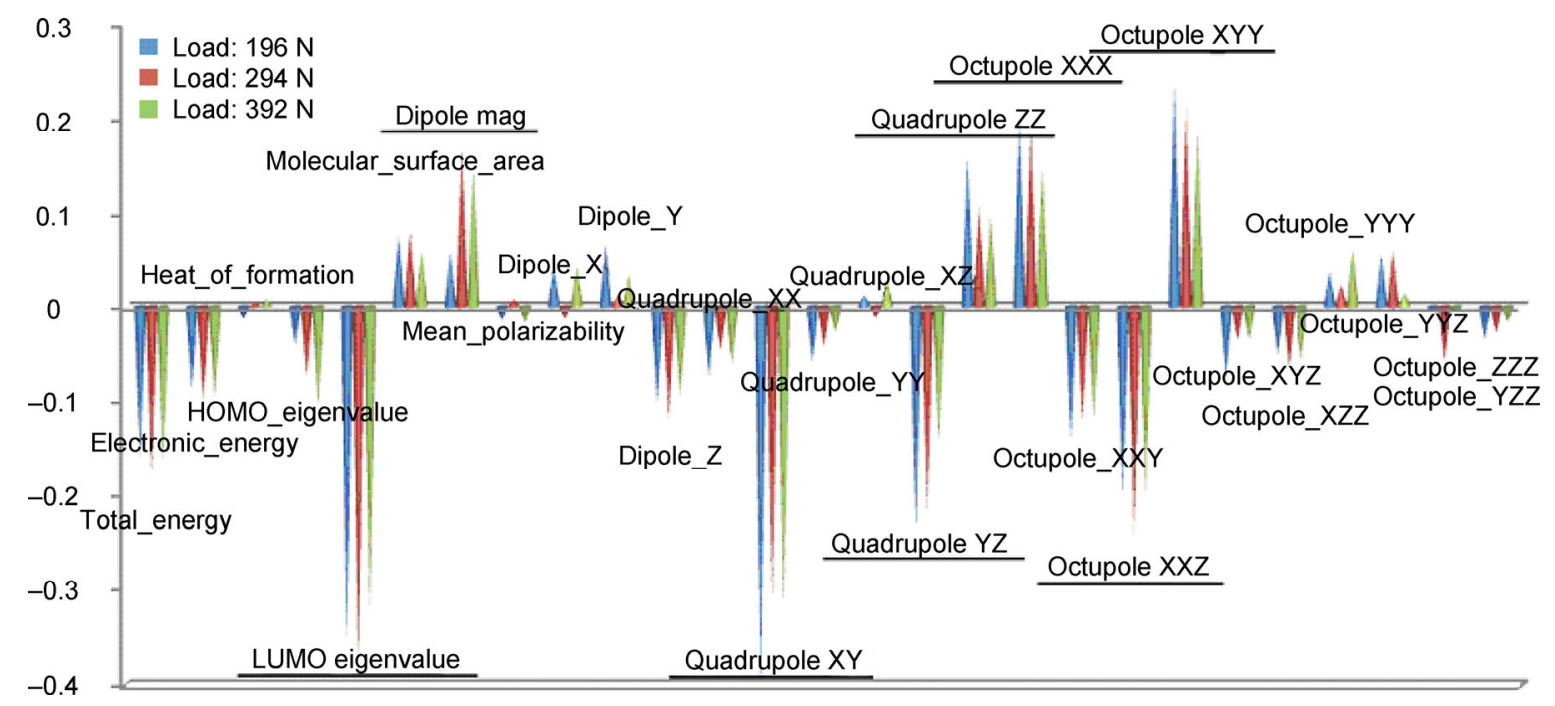

Fig. 4 Sensitivity of quantum descriptors.

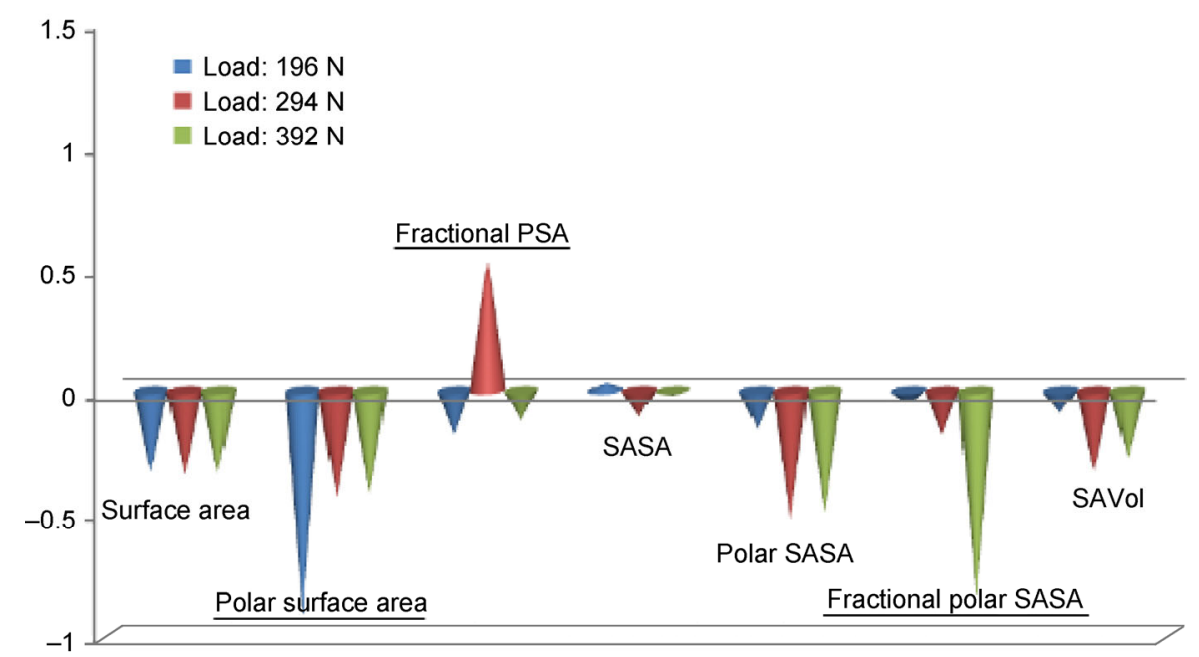

Fig. 5 Sensitivity of the molecular surface area and polar surface area descriptors. 
to optimize the regularization parameter. According to Bayes' theorem

$$
P(w \mid D, M, \alpha, \beta)=\frac{P(D \mid w, \beta, M) P(w \mid \alpha, M)}{P(D \mid \alpha, \beta, M)}
$$

where $P$ is the conditional probability, $D$ is for the sample set, $M$ represents the network model, and $\alpha$ and $\beta$ are the regularization parameters. When the residuals and weighting coefficient are random variables, regularization becomes the extreme value of the random variable. After initially setting $\alpha$ and $\beta$, the minimum value of $F(w)$ is obtained by the LevenbergMarquardt algorithm, $\alpha$ and $\beta$ are updated according to Eq. (4), the optimal value of the posterior distribution is obtained with the highest probability, the new $F(w)$ minimization values are obtained, and then the data are iterated until convergence [18-20].

In summary, the BRNN model fits well in this study. Because the number of descriptors of the QSTR is quite large and significantly more than the number of samples, a multiple linear regression is not applicable, while the PLS method was tested and the results were not satisfactory (data not shown). This is because the lubrication process involves nonlinear processes. The BPNN model uses nonlinear functions and thus fits well to nonlinear processes, making it the best model to analyze friction and wear. In our previous study, we used an ordinary BPNN with the Gaussian-Newton algorithm, but without considering the conditional probability or performing regularization. Therefore, in the process of model building and debugging, the model is prone to over-fitting, and it is timeconsuming to obtain a relatively stable model. The BRNN model proposed here is an improvement to the BPNN model. When modeling, several over-fitting models are excluded; thus, the experimental results are stable and better predict the relationship of the data.

\subsection{D BRNN-QSTR models}

In this study, we found that the 2D BRNN-QSTR model has good predictive and fitting performance, but the other two QSTR models are not good at predicting the antiwear properties of the molecules. The reason for this is that the 3D Jurs and quantum descriptors are associated with a specific molecular conformation. Because we cannot accurately predict the molecular conformation in the lubrication process and the minimum energy conformation may differ from the actual molecular conformation in the lubrication process, the 3D Jurs and quantum descriptors cannot accurately determine the molecular properties in the lubrication process. However, the 2D BRNNQSTR descriptors are classic 2D QSAR (topological) parameters, and the calculation of the 2D QSAR parameters does not involve the molecular conformation, but is closely related to the $2 \mathrm{D}$ molecular structure. This study found that the descriptors of the 2D BRNNQSTR can be used to predict the molecular antiwear performance. In future studies, we will also consider the other 2D QSAR parameters.

\subsection{Sensitivity of descriptor for the antiwear performance}

Each descriptor variable represents a type of molecular structural feature, and here, we focus on the molecular structure of the antiwear performance contribution. The sensitivity of the descriptor variable helps us to examine the contribution of the structural features of the model.

\subsubsection{Sensitivity of quantum descriptors}

Figure 4 shows the sensitivity of the quantum descriptors. In the quantum group, the descriptors of the LUMO show the largest contribution to antiwear. The sensitivities of the LUMO are negative; that is, the lower the energy of the LUMO, the antiwear properties of the molecule are optimal. It can be presumed that the existence of electron transfer processes is similar to chemical reactions in the lubrication process. Electron transfer has an effect on the antiwear process. In previous work [10], we get the result that it is unnecessary to add the aromatic ring system or double bond of compounds to improve the tribological properties, but through the calculation by 2D BRNNQSTR model the result can be amended that it is unnecessary to add the aromatic ring system or double bond of compounds to improve the tribological properties, if the new double bond cannot effect on electron transfer. The other seven influential descriptors 
represent the polarity characteristics of the molecules, in which the dipole moment represents the molecular polarity, and the quadrupole and octupole moments represent not only the orientation of polar molecules but also how the molecules are related to charge in space. It is clear that the molecular charge and the spatial distribution of the electric field have an influence on the process of wear and lubrication.

\subsubsection{Sensitivity of $2 D$ descriptors}

As mentioned in Section 4.2, the 2D QSTR model has the best performance in the BRNN model. A detailed analysis of the 2D structure of the compounds with antiwear properties will be discussed in this section. Forty-seven types of 2D QSTR descriptors were divided into two groups according to their structure: one for the molecular surface area and polar surface area by the 2D method, which includes seven descriptors, in which the sensitivities are shown in Fig. 5; the other group is for the topological descriptors, which include 40 descriptors, in which the sensitivities are shown in Fig. 6(a).

We first analyzed the descriptors for the molecular surface area and the polar surface area (Fig. 5), which were calculated by the $2 \mathrm{D}$ method to obtain the molecular solvation degree [18]. The set of descriptors have different effects on the antiwear process under different load conditions. Polar surface area (PSA), fractional PSA, and fractional polar solvation surface area (fractional polar SASA) have a big effect on antiwear performance. Molecular surface area is the total molecular surface area, which includes the polar and non-polar area; PSA is composed of molecular polar surface area; fractional PSA is the proportion of the molecular polar surface area in the total molecular surface area; and fractional polar SASA is the proportion of the molecular surface area, which may be infiltrated by polar solvent in the total molecular surface area.

In the friction and lubrication process, the additive will diffuse in the base oil and adsorb on the surface of the metal, which will influence the antiwear performance. Almost all of the sensitivities of the descriptors for the molecular surface area and polar surface area are negative under all loads, except for fractional PSA under $294 \mathrm{~N}$ and SASA under $196 \mathrm{~N}$, which are positive.
This means that a high molecular solvation degree of lubricant additive is unfavorable for antiwear, because the adsorption of the lubricant-additive molecules on the metal surface is important for antiwear. Calculation of the molecular polar surface area by different methods can be applied to fit the antiwear process under the different loads. The PSA descriptor is suitable for the low load, the fractional PSA descriptor is suitable for the medium load, and the fractional polar SASA descriptor is suitable for the high load.

The molecular topological descriptors are obtained by analyzing the 2D molecular structure with topological theory. These descriptors represent the structural characteristics of the bonded and nonbonded atoms, and also the substructures, including straight chains, branched chains, and rings, in digital form. Additionally, they provide information about non-carbon atoms (heteroatoms) (see Fig. 6(a)).

Figure 6(b) shows descriptors, including the IAC_ Mean [21], the Balaban indices (JX, JY) [22], and the Wiener index [23]. The IAC_Mean describes the composition of atoms in a molecule, and the presence of heteroatoms will change the IAC_Mean. In the lubricant-additive sample, the IAC_Mean values are positive under all loads, which show that the introduction of heteroatoms is beneficial for the antiwear effect. In these samples, $\mathrm{S}, \mathrm{P}, \mathrm{O}$, and $\mathrm{N}$ are heteroatoms in the lubricant additive, which improve the antiwear performance. However, the inclusion of heteroatoms has minimal effect under the medium load $(294 \mathrm{~N})$ than under the low and high loads. The Balaban indices represent the bonding between the atoms in the molecules. To characterize the influence of the heteroatoms, Balaban indices derived the two descriptors JX and JY: JX is related to the atomic electronegativity; and JY is related to the atomic radius. From Fig. 6(b), the two descriptors are negative under loads of $294 \mathrm{~N}$ and $392 \mathrm{~N}$, and have a significant effect on the antiwear performance under the medium load $(294 \mathrm{~N})$. This demonstrates that elements with low atomic electronegativity and small atomic radius are favored for antiwear performance under medium and high loads.

The Wiener index descriptor describes the bond features of non-hydrogen atoms in the molecule, and does not distinguish between carbon atoms and heteroatoms. Under the medium load, the descriptor 
(a)

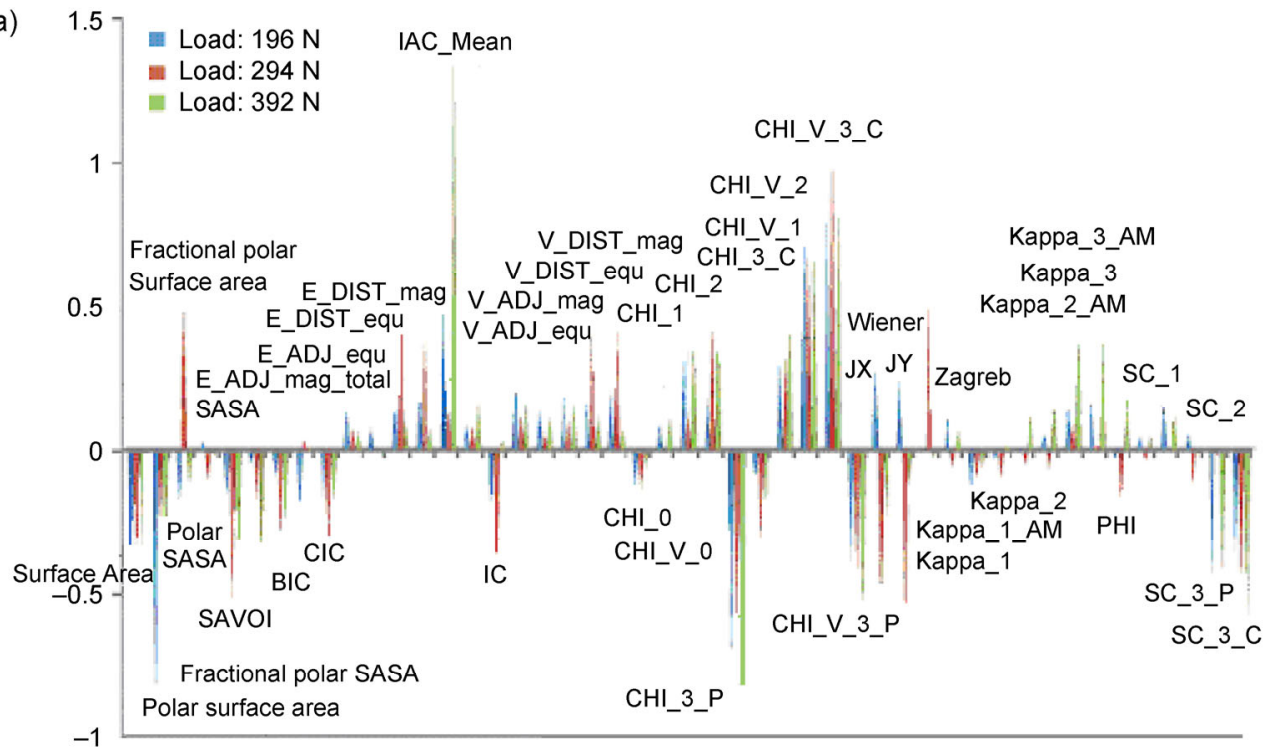

(b)

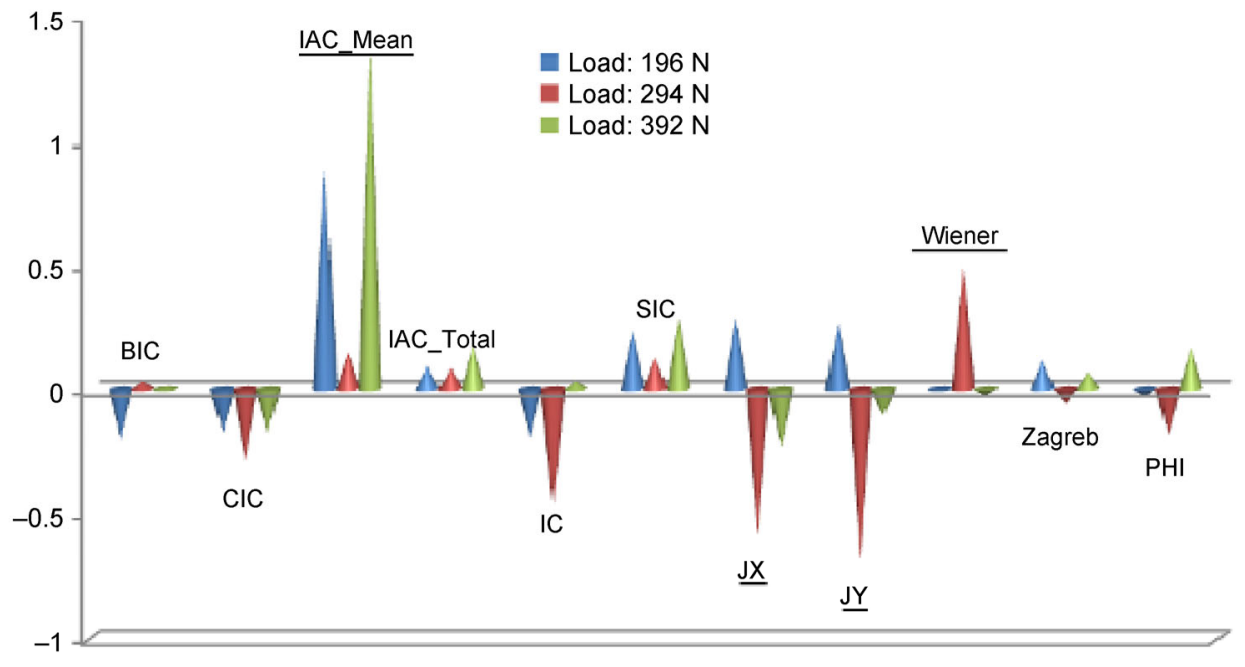

(c)

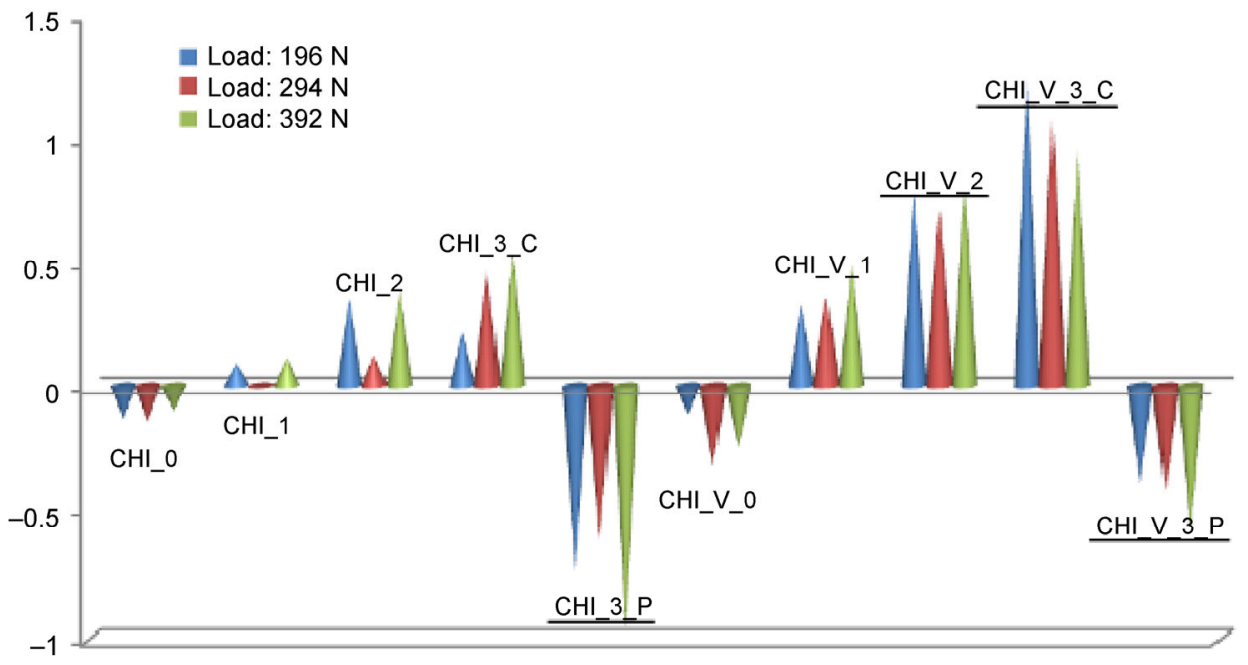

Fig. 6 Sensitivity of topological descriptors. 
is positive and has a large effect on the antiwear, which indicates that stable bonds improve the antiwear effect. This also suggests that under low and high loads the composition of the atoms in molecules, especially the element and the number of heteroatoms, have a large influence on the antiwear properties of the molecules. Presumably, under a low load diffusion and adsorption are important because these processes are related to molecular polarity. Under a high load, the tribological reaction may occur because of electron transfer, which is related to the reactivity of the molecule. Heteroatoms have a large effect on the molecular polarity and reactivity, and thus on the antiwear property of the lubricant additive. Under a medium load, molecular diffusion, adsorption, and the reaction may not be a main influencing factors in the antiwear process. The antiwear process depends on the physical movement of molecules on the metal surface, so the molecular bonding characteristics are likely to be a major factor. Therefore, the Wiener descriptor has a great influence under the medium load.

Figure 6(c) shows the effect of the molecular connectivity indices (CHIs) on the antiwear properties of the molecules. The connectivity indices are important topological descriptors, which are calculated by considering the elements in the molecule and the connection properties between atoms in the substructure [24]. As mentioned above, under high and low loads, the molecular antiwear performance is mainly affected by the atomic composition, especially the heteroatom composition, while under the medium load, the main molecular topology characteristics that affect the antiwear performance are the molecular branched chains and bonding properties. The CHIs combine these two structural characteristics, in which the effect is consistent for all loads. Molecular connectivity indices are difficult to physically interpret, and thus represent a quantitative description of the molecular structure and quantify the structural differences between the molecules. They are easy to calculate, and can predict the physical and chemical properties of compounds without experiments. Therefore, the model provides a better description by using $\mathrm{CHI}$ than other descriptors. This suggests that the structural feature is the primary factor that dominates the antiwear performance of organic compounds. In future work, the relationship between the CHIs and the antiwear property can be further studied.

\section{Conclusions}

We reached the following conclusions from the BRNN-QSTR model:

(1) The BRNN-QSTR model with 2D QSTR descriptors, 3D Jurs descriptors, and quantum descriptors were studied, and therefore, the prediction performance of the 2D BRNN-QSTR model is excellent.

(2) We analyzed the influence of each descriptor on the antiwear property of lubricant additives, and proposed structural characteristics for improving the antiwear ability. The results showed: (a) electron transfer in the lubricant-additive molecule has an effect on the antiwear process; (b) a high molecular solvation degree of lubricant additive is unfavorable for antiwear; (c) heteroatoms, such as S, P, O, and N, improve the antiwear performance; $(\mathrm{d})$ the lubricantadditive molecular bonding characteristics influence the antiwear performance; and (e) CHIs are good descriptors for 2D BRNN-QSTR models.

The QSTR method describes a quantitative relationship between the structure of tribology materials and their tribological properties. This method is a simple, quantitative, and a good predictive model, which provides one way of discovering novel lubricants and exploring the specific mechanisms of the tribology process. The QSTR model reveals the statistical regularity of uncertain phenomena and provides systematic estimation and extrapolation.

\section{Acknowledgments}

This work was supported by the National Basic Research (973) Program of China (No. 2013CB632303) and the National Natural Science Foundation of China (NSFC, No. 51075309). The authors gratefully acknowledge Prof. Junyan Zhang for providing critical data on tribological properties. 
Electronic Supplementary Material: Supplementary material (Compound structures (smiles) and observed and predicted values of the antiwear data in Table S1) is available in the online version of this article at http://dx.doi.org/10.1007/s40544-016-0104-z.

Open Access: The articles published in this journal are distributed under the terms of the Creative Commons Attribution 4.0 International License (http:// creativecommons.org/licenses/by/4.0/), which permits unrestricted use, distribution, and reproduction in any medium, provided you give appropriate credit to the original author(s) and the source, provide a link to the Creative Commons license, and indicate if changes were made.

\section{References}

[1] Liu X Q, Zhou F, Liang Y M, Liu W M. Tribological performance of phosphonium based ionic liquids for an aluminum-on-steel system and opinions on lubrication mechanism. Wear 261(10): 1174-1179 (2006)

[2] Yu G Q, Zhou F, Liu W M, Liang Y M, Yan S Q. Preparation of functional ionic liquids and tribological investigation of their ultra-thin films. Wear 260(9):1076-1080 (2006)

[3] Jiménez A E, Bermúdez M D, Iglesias P, Carrión F J, Martínez-Nicolás G. 1-N-alkyl-3-methylimidazolium ionic liquids as neat lubricants and lubricant additives in steelaluminium contacts. Wear 260(7): 766-782 (2006)

[4] Singh H, Gulati I B. Tribological behaviour of some hydrocarbon compounds and their blends. Wear 139(2): 425-437 (1990)

[5] Martin J M, Grossiord C, Varlo K, Vacher B, Igarashi J. Synergistic effects in binary systems of lubricant additives: A chemical hardness approach. Tribol Lett 8: 193-201 (2000)

[6] Hansch, C., and Steward, A. R.The Use of Substituent Constants in the Analysis of the Structure-Activity Relationship in Penicillin Derivatives. J. Med. Chem., 7: 691-694 (1964)

[7] Li F, Chen J W, Wang Z J, Li J, Qiao X L. Determination and prediction of xenoestrogens by recombinant yeast-based assay and QSAR. Chemosphere 74(9): 1152-1157 (2009)

[8] Tintori C, Magnani M, Schenone S, Botta M. Docking, 3D-QSAR studies and in silico ADME prediction on c-Src tyrosine kinase inhibitors. European Journal of Medicinal Chemistry 44(3): 990-1000 (2009)

[9] Sharma D, Narasimhan B, Kumar P, Jalbout A. Synthesis and QSAR evaluation of 2-(substituted phenyl)-1H-benzimidazoles and [2-(substituted phenyl)-benzimidazol-1-yl]-pyridin-3-ylmethanones. Eur J Med Chem 44(3): 1119-1127 (2009)

[10] Gao X L Wang Z, Zhang H, Dai K. A three dimensional quantitative structure-tribological relationship model. $J$ Tribol 137(2): 021802-1-021802-2 (2015)

[11] Dai K, Gao X L. Estimating antiwear properties of lubricant additives using a quantitative structure tribo-ability relationship model with back propagation neural network. Wear 306(1-2): 242-247 (2013)

[12] Aggarwal K K, Singh Y, Chandra P, Puri M. Bayesian regularization in a neural network model to estimate lines of code using function. J Comput Sci 1: 505-509 (2005)

[13] Zhang J Y. The relationship between additives molecular structure and their tribological properties and the mechanism of boundary lubrication. Ph.D Thesis. Lanzhou: Lanzhou Institute of Chemical Physics, Chinese Academy of Sciences, 1999.

[14] Gao X, Wang Z, Zhang H, Dai K, Wang T. A quantitative structure tribo-ability relationship model for ester lubricant base oils. J Tribol 137(2): 021801-1-021801-7 (2015)

[15] Gao X, Wang R, Wang Z, Dai K. BPNN-QSTR friction model for organic compounds as potential lubricant base oils. J Tribol, in press, doi:10.1115/1.4032304.

[16] Gao X, Liu D, Wang Z, Dai K. Quantitative structure triboability relationship for organic compounds as lubricant base oils using CoMFA and CoMSIA. J Tribol (Accepted)

[17] Gao X L, Dai K, Gao W Z, Wang Z, Wang T T. The application of quantitative structure tribo-ability relationship model. In World Tribology Congress, Turin, Italy, 2013.

[18] MacKay D J C. A practical Bayesian framework for backprop networks. Neural Comput 4(3): 448-472 (1992)

[19] Foresee F D, Hagan M T. Gauss-Newton approximation to Bayesian learning. In Proceedings of the 1997 International Joint Conference on Neural Networks. Houston, 1997: 1930-1935.

[20] MacKay D J C. Bayesian interpolation. Neural Comput 4(3): 415-447 (1992)

[21] Bonchev D. Information Theoretic Indices for Characterization of Chemical Structures, Chemometrics Series. New York: Research Studies Press Ltd, 1983.

[22] Balaban A T. Highly discriminating distance-based topological index. Chem Phys Lett 89: 399-404 (1982)

[23] Müller W R, Szymanski K, Knop J V, Trinajstić N. An algorithm for construction of the molecular distance matrix. J Comput Chem 8(2): 170-173 (1987)

[24] Kier L B, Hall L H. Molecular Connectivity Indices in Chemistry and Drug Research, Medicinal Chemistry. New York: Academic Press, 1976. 


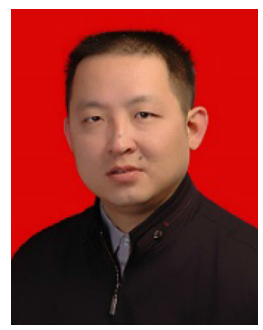

Kang DAI. He received his M.S. and Ph.D. in medicinal chemistry from Tongji Medical College, Huazhong University of Science and Technology, Wuhan, China in

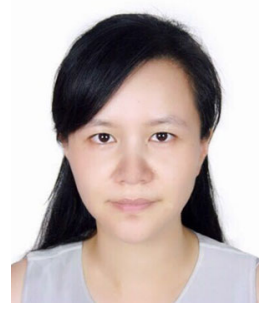

Xinei GAO. She received her M.S. degree in 1996 from Huazhong Normal University in organic chemistry, and graduated from Wuhan Research Institute of Materials Protection in mechanical design
1997 and 2002 respectively. He joined South-Central University for Nationalities from 2005. His research interests include pharmaceuticals and computer aided drug design.

and theory with Ph.D. degree in 2006. Currently she is a full professor at Wuhan Polytechnic University, member of Chinese Tribology Association. She is interested in tribology chemistry, chemical computing, and designation of lubricant. 